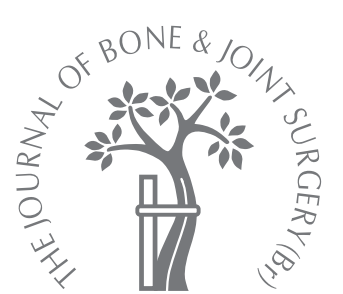

R. C. I. van Geenen, P. P. Besselaar

From the Academic

Medical Centre, Amsterdam, The Netherlands

\title{
Outcome after corrective osteotomy for malunited fractures of the forearm sustained in childhood
}

\begin{abstract}
We analysed the operative technique, morbidity and functional outcome of osteotomy and plate fixation for malunited fractures of the forearm sustained in childhood.

A total of 20 consecutive patients underwent corrective osteotomy of 21 malunited fractures at a mean age of 12 years ( 4 to 25). The mean time between the injury and the osteotomy was 30 months (2 to 140 ).

After removal of the plate, one patient suffered transient dysaesthesia of the superficial radial nerve. The mean gain in the range of movement was $85^{\circ}\left(20^{\circ}\right.$ to $\left.140^{\circ}\right)$. The interval between injury and osteotomy, and the age at osteotomy significantly influenced the functional outcome ( $p=0.011$ and $p=0.004$, respectively).

Malunited fractures of the forearm sustained in childhood can be adequately treated by osteotomy and plate fixation with excellent functional results and minimal complications. In the case of established malunion it is advisable to perform corrective osteotomy without delay.
\end{abstract}

Fractures of the forearm in children are managed routinely by closed methods. There is little agreement as to how much angulation is acceptable according to age. Some advise that all displaced fractures in patients over 14 years of age should be treated by operative fixation ${ }^{1}$ whereas others recommend an age limit of eight years. ${ }^{2}$ The cut-off value of angulation as a requirement for surgical correction varies from $15^{\circ}$ in mid-shaft fractures ${ }^{3}$ to $40^{\circ}$ in fractures of the distal third. ${ }^{4}$

Closed treatment of diaphyseal fractures carries the risk of malunion. Angulation, displacement and malrotation may cause malunion with a rotational deficit. The more proximal the location of the malunion, the more pronounced the resulting deficit, ${ }^{5-8}$ because spontaneous correction only occurs in the distal metaphysis if sufficient growth remains ${ }^{2}$ and periosteal remodelling can only play a significant role in early childhood.

Malunion with forearm rotation of less than $50 \%$ to $60 \%$ of normal is associated with considerable functional impairment, ${ }^{9,10}$ and justifies corrective osteotomy. The operation may also be indicated in patients with a smaller rotational deficit but who require a high range of rotation for good function.

The outcome of corrective osteotomy appears to depend on the morbidity of the operation and the interval between the initial injury and the osteotomy. ${ }^{11}$ We have analysed the morbidity and functional outcome of open corrective osteotomy for malunited fractures of the forearm in children, and identified factors which influence the functional outcome.

\section{Patients and Methods}

Between 1986 and 2005, 20 consecutive children (21 fractures) underwent corrective osteotomy for a malunited fractures of the forearm. Patients who sustained a fracture after the age of 16 years were excluded. One girl had bilateral malunited forearm fractures. There were 12 boys and eight girls with a mean age of ten years (2 to 15$)$ at the time of injury. In 13 patients both the radius and ulna were fractured and in eight the radius only. The fractures were in the proximal third in nine, middle third in seven, and in the distal third in five. A total of 18 fractures had been treated initially by closed reduction and three by operative procedures. One patient who was previously treated with a Kirschner $(\mathrm{K})$-wire fixation of the radius underwent a corrective osteotomy for malunion before referral which resulted in another malunion. The mean time between the initial injury and osteotomy was 30 months (2 to 140 ) and the mean age at osteotomy was 12 years (4 to 25 ).

Pre-operative assessment included documentation of previous treatment and measure- 


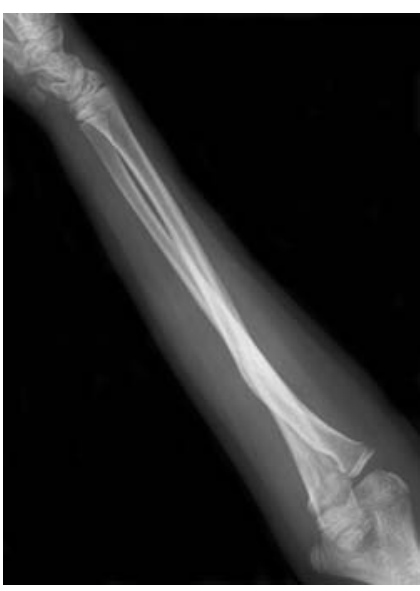

Fig. 1a

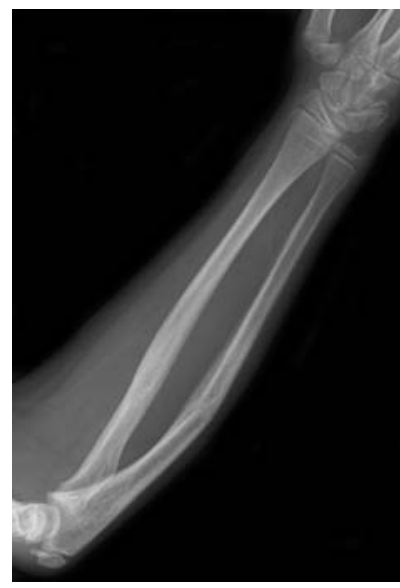

Fig. 1b
Malunion of the right forearm with angulation in an eight-year-old girl, a) anteroposterior and b) lateral radiographs of malunion eight months after the initial trauma, before corrective osteotomy.

ment of the carrying angle, angulation and rotation of the forearm, flexion and extension of the elbow and the wrist, and stability of the proximal and distal radioulnar joints. Full-length anteroposterior and lateral radiographs were taken (Fig. 1). The humeral epicondyles served as a reference point for obtaining two perpendicular projections. The forearm was held in neutral rotation because pronation and/or supination were compromised. If possible, comparison radiographs of the unaffected arm were taken. The plane of maximum angulation was identified under fluoroscopic guidance. Pre-operative planning of the tri-plane osteotomy was essential and was based on careful analysis of all the findings.

Operative technique. The radius is usually exposed first through an anterior approach, because restoring the site and amount of radial bowing is of great importance in optimising rotation. ${ }^{10}$ Care is taken to avoid damage to the deep and superficial radial nerves and the radial vascular bundle. The position of maximal angulation is marked and $\mathrm{K}$-wires are placed proximal and distal to the proposed site of the osteotomy at sufficient distance to mark rotation. Depending on the diameter of the diaphysis, a $2.7 \mathrm{~mm}$ or $3.5 \mathrm{~mm}$ dynamic compression plate is used and temporarily fixed by a proximal screw. The site of the osteotomy is marked and the plate removed. After subperiosteal exposure, a transverse osteotomy is undertaken. The radial bow is restored and a wedge resection performed to correct angulation. The plate is bent to fit the restored radial bow and held by the proximal screw and a bone clamp distally. Pronation and supination are tested and the reduction and radial bow checked with an image intensifier.

If necessary, the ulna is exposed through an incision parallel to the ulnar crest and osteotomised in a similar fashion to restore alignment. After temporary fixation, pronation and supination are checked and when sufficient, definitive fixation of both bones is performed.

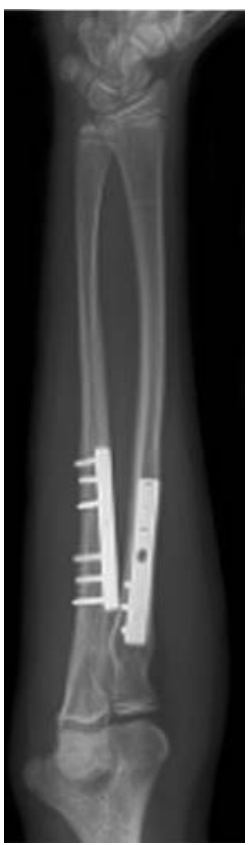

Fig. $2 a$

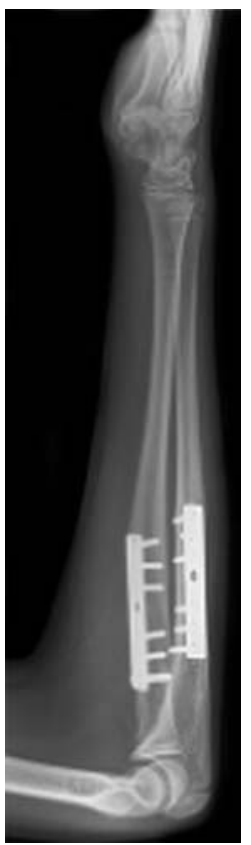

Fig. $2 b$ a) Anteroposterior and b) lateral radiographs of the same patient as in Figure 1 two years after corrective osteotomy and plating.

A well-padded above-elbow splint is applied and the patient allowed to exercise on a continuous passivemovement machine under the supervision of a physiotherapist from the first post-operative day. Radiographs are taken at six and 12 weeks and at six and 12 months. The plates are removed when remodelling is complete and after a minimum of 1.5 years to avoid refracture (Fig. 2).

Post-operative complications, re-operations and pre- and post-operative ranges of movement were analysed. Factors which might influence outcome were analysed, namely the age at injury, the fractured bones (radius and/or ulna), the level of fracture (proximal, middle or distal third), the osteotomies (radius and/or ulna) and the time from injury to osteotomy (more or less than one year).

Statistical analysis. Non-parametric statistical analysis was performed using SPSS program V11.5 (SPSS Inc., Chicago, Illinois) since sample sizes were small. The Mann-Whitney test was used to compare the gain in the range of movement for different factors. A p-value $\leq 0.05$ was considered to be statistically significant.

\section{Results}

In ten cases both bones underwent osteotomy, and in 11, osteotomy of the radius was sufficient. In six of 11 cases requiring isolated radial osteotomy the radius alone had been fractured at original presentation with minimal or no bowing of the ulna. In one case the ulna had previously been fixed internally. In four children in whom both bones 
Table I. Factors influencing the gain in the range of movement

\begin{tabular}{|c|c|c|c|c|}
\hline Factors & Number of patients ${ }^{*}$ & $\Delta \mathbf{R O M}^{\dagger}$ & Range & p-value \\
\hline \multicolumn{5}{|l|}{ Age at initial injury (yrs) } \\
\hline$<10$ & 9 & 92 & 60 to 115 & 0.186 \\
\hline$\geq 10$ & 12 & 72 & 20 to 140 & \\
\hline \multicolumn{5}{|l|}{ Bones fractured } \\
\hline Radius & 8 & 88 & 65 to 115 & 0.662 \\
\hline Radius and ulna & 13 & 83 & 20 to 40 & \\
\hline \multicolumn{5}{|l|}{ Fracture level } \\
\hline Proximal third & 9 & 85 & 65 to 110 & 0.886 \\
\hline Distal or middle third & 12 & 84 & 20 to 140 & \\
\hline \multicolumn{5}{|c|}{ Time between injury and osteotomy (yrs) } \\
\hline$<1$ & 8 & 98 & 20 to 140 & 0.011 \\
\hline$\geq 1$ & 13 & 76 & 60 to 100 & \\
\hline \multicolumn{5}{|l|}{ Age at osteotomy (yrs) } \\
\hline$<10$ & 6 & 105 & 95 to 115 & 0.004 \\
\hline$\geq 10$ & 16 & 76 & 20 to 140 & \\
\hline \multicolumn{5}{|l|}{ Bones osteotomised } \\
\hline Radius & 11 & 82 & 60 to 115 & 0.457 \\
\hline Radius and ulna & 10 & 87 & 20 to 140 & \\
\hline
\end{tabular}

were involved, the deformity was predominantly in a malunited radius and was corrected adequately by osteotomy of the radius. Bone grafting was not required in any patient. The rehabilitation programme preserved the intraoperative rotational gain, which did not improve further after removal of the plate. In six children the plates were in situ at follow-up.

After removal of the plate, one patient suffered transient dysaesthesia of the superficial radial nerve. One patient (case 3 ) sustained a traumatic fracture of the radius at the distal end of the plate and was treated by closed reduction and immobilisation. This patient died during follow-up from co-existing cirrhosis and portal hypertension. Since this fracture occurred in a patient with a severe systemic disease and followed significant trauma, it was not regarded as a complication of the osteotomy.

The mean pre-operative pronation was $26^{\circ}\left(0^{\circ}\right.$ to $\left.90^{\circ}\right)$, supination was $13^{\circ}\left(-60^{\circ}\right.$ to $\left.+70^{\circ}\right)$ and the mean range of movement $38^{\circ}\left(0^{\circ}\right.$ to $\left.90^{\circ}\right)$. The mean follow-up period was 23 months (4 to 59). At the last follow-up the mean pronation was $60^{\circ}\left(10^{\circ}\right.$ to $\left.85^{\circ}\right)$, supination was $63^{\circ}\left(10^{\circ}\right.$ to $\left.90^{\circ}\right)$, and the mean range of movement in pronation/supination was $124^{\circ}\left(20^{\circ}\right.$ to $\left.165^{\circ}\right)$. The mean gain in the range of movement was $85^{\circ}\left(20^{\circ}\right.$ to $\left.140^{\circ}\right)$. No patient had a decrease in the range of movement.

The corrective osteotomy was performed within one year after injury in eight fractures and after one year in 13 . The former group had a significantly higher gain in range of movement than the latter $(\mathrm{p}=0.011)$. Those children under ten years of age at osteotomy had a significantly higher gain in the range of movement compared with those over ten years of age at osteotomy $(\mathrm{p}=0.004$; Table I). The age at injury, the fractured bones, the level of the fracture and the bones osteotomised did not influence the gain in the range of movement (Table I).

\section{Discussion}

Most children have an uneventful recovery after a fracture involving the forearm. However, malunion may occur in $15 \%^{2}$ and a rate of malunion of $35 \%$ has been reported after fractures which had been mostly treated conservatively. ${ }^{12}$ Since malunion can result in considerable functional loss, pain, instability and cosmetic changes, corrective osteotomy is required and a more aggressive attitude towards primary treatment has been advocated. ${ }^{12}$

Our technique for osteotomy and fixation has proved to be reliable and without complication. After removal of the plate only one patient had transient dysaesthesia, which was considerably lower than the rate of $37 \%$ found in a study using a comparable technique. ${ }^{11}$ This may be explained partially by the higher mean age at osteotomy of 19 years in that study compared with 12 years in ours. Children are less likely to suffer from infection or nonunion. However, complications such as heterotopic ossification and damage to the radial nerve can be related to the wide exposure and therefore other techniques such as drill osteoclasis have been developed. ${ }^{13}$ In this method the convex cortex is weakened by drill holes through a stab incision and, after closed manipulation, a long-arm cast is applied. The risks of a wide exposure are thereby avoided and only two refractures in patients with osteogenesis imperfecta have been described, with no other complications reported. Although the technique can correct angulation, it is difficult to apply accurately and to maintain angular correction. Also, it is impossible to correct rotation. Therefore, we believe that open reduction and plating is the preferred technique. 
The ability for spontaneous correction of angulation decreases with advancing age. In our series the age at injury varied from two to 15 years. All the patients had limited rotation which did not correct spontaneously and required an osteotomy. This included six children aged eight years or less, including one child with bilateral fractures. Others have advised operative alignment only for children of eight years or more. ${ }^{2}$ This suggests that the mechanism of spontaneous correction of malunited forearm fractures may be overestimated.

The site of the fracture can affect functional outcome. The more proximally it is located, the greater is the risk of angulation and rotation malunion..$^{5-8}$ In our series, only five patients had a distal fracture. However, the location of the fracture did not influence the outcome after osteotomy because patients with malunion after a proximal fracture had a comparable gain in rotation $\left(85^{\circ}\right)$ compared with those undergoing osteotomy in the middle or distal third of the forearm $(\mathrm{p}=0.886)$.

In our series, the effect of the interval between the initial injury and osteotomy influenced post-operative function. The gain in the range of movement was greater in the eight malunions treated within one year. This finding agrees with that of an earlier report in which nine patients were treated within one year and gained a mean of $79^{\circ}\left(20^{\circ}\right.$ to $\left.160^{\circ}\right) \mathrm{com}-$ pared with $30^{\circ}\left(-25^{\circ}\right.$ to $\left.+95^{\circ}\right)$ in 11 patients treated after one year. ${ }^{11}$ This effect may have been due to soft-tissue contraction after the initial trauma and to secondary changes in the joints. Therefore it is recommended that malunion is corrected before changes occur.

Our findings suggest that remodelling could positively affect the functional outcome in patients who underwent an osteotomy at a younger age. Children undergoing a corrective osteotomy before the age of ten years had a significantly greater range of movement than those over ten years. However, this effect might also be explained by the high similarity in age at osteotomy and the interval between injury and osteotomy. The numbers involved are too small to allow multivariate analysis but we believe it is highly likely that the time between the initial injury and the osteotomy is more important.

A single osteotomy is not always sufficient to correct rotational loss and both bones may need osteotomies. We thought that in such cases the gain in movement would be less compared with that in a single osteotomy. However, our data did not support this. Even complex malunions could be adequately realigned with a sufficient gain in the range of movement.

In conclusion, malunited fractures of the forearm which are sustained in childhood can be treated satisfactorily by open reduction and plate fixation. Our technique gave excellent functional results with few complications. It is advisable to perform corrective osteotomy at the earliest opportunity. It remains unclear whether the age at osteotomy independently influences the outcome.

\section{Supplementary Material}

苑 A supplementary table showing the details of the 20 children and their treatment is available with the electronic version of this article on our website at www.jbjs.org.uk

No benefits in any form have been received or will be received from a commercial party related directly or indirectly to the subject of this article.

\section{References}

1. Hughston JC. Fractures of the forearm in children. J Bone Joint Surg [Am] 1962;44-A:1678-93.

2. Fuller DJ, McCullough CJ. Malunited fractures of the forearm in children. $J$ Bone Joint Surg [Br] 1982;64-B:364-7.

3. Daruwalla JS. A study of radioulnar movements following fractures of the forearm in children. Clin Orthop 1979;139:114-20.

4. Cooper RR. Management of common forearm fractures in children. J lowa Med Soc 1964;54:689-98

5. Matthews LS, Kaufer H, Garver DF, Sonstegard DA. The effect on supination-pronation of angular malalignment of fractures of both bones of the forearm. J Bone Joint Surg [Am] 1982;64:14-17.

6. Tarr RR, Garfinkel Al, Sarmiento A. The effects of angular and rotational deformities of both bones of the forearm: an in vitro study. J Bone Joint Surg [Am] 1984;66-A:65-70.

7. Weinberg AM, Pietsch IT, Krefft M, et al. Pronation and supination of the forearm: with special reference to the humero-ulnar articulation. Unfallchirurg 2001;104:404-9 (in German).

8. Roberts JA. Angulation of the radius in children's fractures. J Bone Joint Surg [Br] 1986;68-B:751-4

9. Anderson LD, Sisk D, Tooms RE, Park WI III. Compression-plate fixation in acute diaphyseal fractures of the radius and ulna. J Bone Joint Surg [Am] 1975:57-A:287-7.

10. Schemitsch EH, Richards RR. The effect of malunion on functional outcome after plate fixation of fractures of both bones of the forearm in adults. J Bone Joint Surg [Am] 1992;74-A:1068-78.

11. Trousdale RT, Linscheid RL. Operative treatment of malunited fractures of the forearm. J Bone Joint Surg [Am] 1995;77-A:894-902.

12. Schmittenbecher PP. State-of-the-art treatment of forearm shaft fractures. Injury 2005;36(Suppl 1):25-34.

13. Blackburn N, Ziv I, Rang M. Correction of the malunited forearm fracture. Clin Orthop 1984;188:54-7. 